\title{
Factors related to preventive COVID-19 behaviors using health belief model among general population: a cross-sectional study in Iran
}

Mahmood Karimy ${ }^{1}$, Fatemeh Bastami ${ }^{2}$, Robab Sharifat ${ }^{3}$, Akbar Babaei Heydarabadi ${ }^{4}$, Naser Hatamzadeh ${ }^{4}$, Amir H. Pakpour ${ }^{5,6}$, Bahman Cheraghian ${ }^{7}$, Fereshteh Zamani-Alavijeh ${ }^{8}$, Mehrnoosh Jasemzadeh ${ }^{4}$ and Marzieh Araban $^{4^{*}}$ (1)

\begin{abstract}
Background: Coronavirus disease (COVID-19) pandemic has become one of the biggest challenges to global health and economy. The present study aimed to explore the factors related to preventive health behaviors during the COVID-19 pandemic in Khuzestan Province, South of Iran, using the Health Belief Model (HBM).

Methods: The present cross-sectional study was conducted in the period between July 2020 and September 2020. A total of 1090 people from Khuzestan province participated in the study. The data collection method included a multistage cluster sampling method with a random selection of provincial of health centers. The questionnaire collected socio-demographic information and HBM constructs (e.g., perceived susceptibility, perceived severity, perceived benefits and barriers, cues to action, and COVID-19 preventive behaviors). Data were analyzed using ANOVA, t-test, hierarchical multiple linear regression, and SPSS version 22.

Results: The mean age of the participants was $35.53 \pm 11.53$, more than half of them were female (61.6\%) and married (65.3). The results showed that 27\% of the variance in the COVID-19 preventive behaviors was explained by HBM constructs. The regression analysis indicated that female gender $(\beta=0.11)$, perceived benefits $(\beta=0.10)$, perceived barriers $(\beta=-0.18)$, external cues to action $(\beta=0.25)$, and internal cues to action $(\beta=0.12)$ were significantly associated with COVID-19 preventive behaviors $(p<0.05)$.

Conclusion: Designing an educational intervention on the basis of HBM might be considered as a framework for the correction of beliefs and adherence to COVID-19 behavior. Health information campaigns need to (1) emphasize the benefits of preventive behaviors including avoiding the likelihood of getting a chronic disease and complications of the disease, (2) highlight the tips and advice to overcome the barriers (3) provide cues to action by means of showing various reminders in social media (4) focusing on adoption of COVID-19-related preventive behaviors, especially among men.
\end{abstract}

Keywords: COVID-19, Preventive behavior, Health belief model, Iran

\footnotetext{
* Correspondence: araban62@gmail.com; arabanm@ajum.ac.ir

${ }^{4}$ Department of Health Education and Promotion, School of Public Health,

Ahvaz Jundishapur University of Medical Sciences, Ahvaz, Iran

Full list of author information is available at the end of the article
}

(c) The Author(s). 2021 Open Access This article is licensed under a Creative Commons Attribution 4.0 International License, which permits use, sharing, adaptation, distribution and reproduction in any medium or format, as long as you give appropriate credit to the original author(s) and the source, provide a link to the Creative Commons licence, and indicate if changes were made. The images or other third party material in this article are included in the article's Creative Commons licence, unless indicated otherwise in a credit line to the material. If material is not included in the article's Creative Commons licence and your intended use is not permitted by statutory regulation or exceeds the permitted use, you will need to obtain permission directly from the copyright holder. To view a copy of this licence, visit http://creativecommons.org/licenses/by/4.0/ The Creative Commons Public Domain Dedication waiver (http://creativecommons.org/publicdomain/zero/1.0/) applies to the data made available in this article, unless otherwise stated in a credit line to the data. 


\section{Background}

The coronavirus disease 2019 (COVID-19) pneumonia pandemic is a new emerging global challenge in the management of infectious diseases [1-4]. At present, the disease has spread rapidly and affected the whole world with epidemiological features such as rapid transmission, increasing prevalence in a short period of time, extensive incubation period (2-14 days), as well as the ability to infect all individuals and groups [5].

A number of measures have been developed to prevent and mitigate the transmission and mortality associated with COVID-19 including rapid identification of suspected cases, rapid testing and isolation, contact tracing and quarantine, restrictions on non-essential domestic and international travel, and high-level regular participation of communities [6]. Currently, the transmission routes of COVID-19 infection are well recognized. Governments and other sectors such as media, health workers, celebrities, police, and other stakeholders have focused primarily on behaviors that prevent the transmission of the virus, such as wearing masks, keeping physical distance, hand-washing practices, and avoiding public meetings (such as religious ceremonies and family gatherings) $[7,8]$. Despite taking preventive programs, the rate of COVID-19 infection remains high, indicating that these programs have not been effective in controlling the infection $[9,10]$. For instance, a study by Smith et al. [11] among adolescents aged 12-15 years in 80 countries showed that the prevalence of never/rarely hand washing practices before eating and after using the toilet was 6.4 , and $5.6 \%$, respectively.

In Iran, previous studies have shown that despite the recommendations of international, national, and regional organizations, the level of behavioral adherence to prevent coronavirus in the general population is still unsatisfactory [9]. Therefore, it is necessary to identify factors affecting adherence to recommended guidelines. Behavioral models are designed to identify factors that influence behavior change in order to control the infection [12]. Various models/theories have been proposed to identify factors affecting behavior change. The Health belief model $(\mathrm{HBM})$ is a comprehensive model in health-behavioral sciences [13]. Previous studies have supported the HBM as a useful model for predicting/ explaining preventive behaviors against infectious diseases such as COVID-19 $[8,13]$.

HBM is a model that focuses on individual beliefs about health conditions [7]. According to this model, the probability that a person participates in a health practice is based on individual beliefs; the probability of adopting recommended behaviors (e.g. preventive COVID-19 behaviors) will increase by changing the individual perceptions. According to the health belief model, people will adopt preventive health behavior when they feel threatened by pandemic situations (perceived susceptibility) or consider that the disease can have serious ramifications to their health (perceived severity). Likewise, with the information and guidance people receive from their surroundings or inner environment (cues to action), they believe in the usefulness of preventive behaviors such as using a mask (perceived benefits), and the perception of negative aspects (costs) of a given behavior to perform (perceived barriers) [13, 14].

According to the World Health Organization (WHO) report (June 06, 2021), Iran had 2,966,363 COVID-19 cases [15]. Khuzestan province, southwest of Iran, is among the regions with the highest number of COVID19 cases [16]. Considering the importance of preventive behaviors in reducing the transmission of COVID-19, the present study aimed to explore the factors related to preventive health behaviors during the COVID-19 pandemic in Khuzestan province using the Health Belief Model (HBM).

\section{Methods \\ Study design and participants}

The present cross-sectional study was conducted in the period between July 2020 and September 2020. A total of 1090 citizens from the catchment area of Khuzestan province participated in the study. The inclusion criteria were as follows: 15 years of age or older, ability to communicate in Persian, living in Khuzestan province, and voluntary consent to participate in this study.

\section{Sampling}

A multistage cluster sampling method was adopted for the recruitment of study participants. The counties of Khuzestan province, Ahvaz County (the capital of Khuzestan province) and five other counties (clusters) including Omidiyeh, Izeh, Shush, Hoveyzeh, and Dasht-e Azadegan were selected as a cluster unit. Within each county (cluster) there were two elements (urban and rural). For sampling of urban areas, a health center from the downtown area and one health center from the south of city were randomly selected using a map of the population area. In rural areas, the potential list of the population was prepared by a health care provider who was not a member of the study group. Then, a systematic random sampling technique with a sampling interval of three was employed to select the study participants. The selected geographic location in the map was not limited to any specific geographic region. Then, within the selected urban and rural health centers, based on the file number of households, systematic sampling with probability proportional to size was applied to select the households, i.e., the larger health center population density, the higher the share of the total sample size. In the final stage, two individuals (preferably a man and a 
woman) were sampled within each household. "The final sample was 1,090": (Ahvaz $=425$, Omidieh $=369$, Izeh $=$ 86 , Shush $=107$, Hoveyzeh $=71$, and Dasht-e Azadegan $=$ 32).

\section{Measures}

A three-part self-report -researcher-made- questionnaire (Additional file 1) which relied on demographic, HBM constructs, and preventive behaviors items was used to collect data.

\section{Demographic sheet}

The first section of the questionnaire included demographic characteristics including age, sex, education, occupation, and history of chronic diseases.

\section{HBM constructs}

The second section of the questionnaire included questions on HBM structures as follows: 1- Perceived susceptibility (7 items); 2- Perceived severity (5 items); 3Perceived benefit (4 items); 4- Perceived barrier (5 items); 5- Internal cues to action (4 items); and 6- External cues to action ( 4 items). All items were rated on a five-point Likert type scale ranging from strongly disagree (1) to strongly agree (5), (Table 1 ).

\section{Preventive behaviors}

The third part of the questionnaire included questions concerning the preventive COVID-19 behavior of the individual during the last month. This part was designed based on similar studies [17-20]. Seven items were used to measure preventive COVID-19 behavior: The behavior questions included self-report items such as: Do you wash your hands for $20 \mathrm{~s}$ after touching surfaces or outdoor items? Do you use a mask in public places? Do you refuse to go to family ceremonies (celebrations, funerals, parties, etc.)? Do you cover your mouth when sneezing or coughing? Do you disinfect surfaces with disinfectants? Do you adhere to the principles of social distancing? Do you disinfect commonly used surfaces and equipment with disinfectants? The rating scale was on a five-point scale: never $=1, \quad$ rarely $=2, \quad$ sometimes $=3$, often $=4$, always $=5$. The possible range of scores was
7-35. The internal consistency was acceptable as $\alpha=$ 0.89 .

\section{Validity \& reliability}

The validity of the present questionnaire was determined by face and content validity methods. The face validity of the items (quantitative and qualitative (was determined by 20 participants and calculated using the impact score equation) Impact Score $=$ Frequency $(\%) \times$ Importance). Questions with an impact score higher than 1.5 were considered acceptable. At this stage, two questions were deleted. The qualitative content validity of this questionnaire was observed and evaluated by 10 infectious disease and public health specialists. The content validity ratio (CVR) was determined using the Lawshe table (CVR values of higher than 0.75). The specialists were enquired to stipulate whether an item is necessary for running a construct in a set of items. The specialists were requested to rate each item according to the Content Validity Index (CVI), relevance, clarity, and simplicity (CVI value 0.79 was considered acceptable) [21]. The internal consistency reliability of all constructs was found to be satisfactory $(\alpha>0.70$, Table 1$)$.

\section{Sample size}

The sample size was determined according to the following formula based on the data obtained from a pilot study in which the smallest correlation was considered the largest sample size. $\mathrm{n}=\left[\frac{\mathrm{Z} 1-\alpha / 2+\mathrm{Z} 1-\mathrm{B}}{0.5 \operatorname{Ln}\left(\frac{1+r}{1-r}\right)}\right]^{2}+3 ; \mathrm{d}=0.05$, $\beta=0.2, r=0.12$. Finally, the sample size was estimated to be 540 people. The final sample size was determined to be 1080 people (considering design effect equal to 2). A total of 1100 questionnaire sheets were distributed to the participants to obtain the required 1080 .

\section{Data analysis}

Inferential statistics (independent t-test and one-way analysis of variance) were used for comparisons between groups. Correlation and multiple hierarchical linear regressions were performed to test the relationships between HBM and the health-related behaviors. To explore the factors affecting preventive behaviors, bivariate analysis was performed with demographic variables

Table 1 Description of the HBM constructs

\begin{tabular}{|c|c|c|c|c|}
\hline HBM constructs & number of items & score ranges & Cronbach's alpha & McDonald's $\omega$ \\
\hline Perceived susceptibility & 7 & $7-35$ & 0.72 & 0.78 \\
\hline Perceived severity & 5 & $5-25$ & 0.70 & 0.72 \\
\hline Perceived benefits & 4 & $4-20$ & 0.78 & 0.78 \\
\hline Perceived barriers & 5 & $5-25$ & 0.85 & 0.86 \\
\hline internal cues to action & 4 & $4-20$ & 0.75 & 0.75 \\
\hline External cues to action & 4 & $4-20$ & 0.87 & 0.87 \\
\hline
\end{tabular}


and HBM constructs, of which, gender, education (the number of years of formal education), occupation, marital status, and HBM constructs were significantly associated with behavior. In the next step, variables associated with the outcome in the bivariate analysis with $p$-value $<0.25$ were entered in the model. An analysis of residuals confirmed the assumptions of linearity. It should be mentioned that collinearity was checked and was negative. Data were analyzed using SPSS software Version 22.0 (SPSS Inc., Chicago, IL, USA). P-value less than 0.05 at the final stage was considered statistically significant.

\section{Ethical considerations}

The study was approved by the Ethics Committee of Ahvaz Jundishapur University of Medical Sciences, Ahvaz, Iran (Registration No: IR.AJUMS.REC.1399.145). Written informed consent was obtained from the participants. During data collection, social distancing was maintained and in the period of collecting the responses, both the respondent and the research assistants were wearing face masks.

\section{Results}

Among the 1100 distributed questionnaires, 10 questionnaires were excluded from the analysis owing to incomplete and distorted information.

The mean age of the participants was $35.53 \pm 11.53$ years, the majority of them were women $(61.6 \%)$ and married (65.3\%). The participants' level of education ranged from high school (36.8\%) to university education $(30.8 \%)$. In the case of men, $31.9 \%$ were self-employed and $29.3 \%$ were employees. The highest percentage of occupation in women was 66.6\% (homemaker (, 19.3\% (Employee), and 7.3\% (self-employment), (Table 2).

More than two-thirds of the participants (76.3\%) reported that they had no chronic disease. Hypertension (5.5\%) and diabetes (5\%) were the most prevalent selfreported doctor-diagnosed chronic conditions.

There was a positive and significant correlation between HBM constructs and COVID-19 preventive behaviors (external cues to action $(r=.47)$, internal cues to action $(r=.42)$, perceived benefits $(r=.30)$, and perceived severity $(r=.21)$. A negative correlation was found between perceived barriers $(r=-.20)$ and COVID-19 preventive behaviors $(p<0.05$, Table 3$)$.

Table 4 shows that the perceived severity of COVID-19 among older people (aged +70 years) was more than that of younger people (aged 15-29). A significant correlation was found between constructs of cues to action and preventive behaviors $(\mathrm{p}<0.05)$. Also, women had better preventive behaviors than men. Similarly, people with university-level education compared to the elementary level, married people compared to single people, and
Table 2 Descriptive statistics of demographic variables

\begin{tabular}{|c|c|c|}
\hline \multicolumn{3}{|l|}{ Variables } \\
\hline Age & $\mathbf{N}$ & $\%$ \\
\hline $15-29$ & 320 & 29.4 \\
\hline $30-49$ & 530 & 48.6 \\
\hline $50-69$ & 172 & 15.7 \\
\hline$\geq 70$ & 68 & 6.2 \\
\hline \multicolumn{3}{|l|}{ Gender } \\
\hline Male & 419 & 38.5 \\
\hline Female & 671 & 61.5 \\
\hline \multicolumn{3}{|l|}{ Education } \\
\hline University & 336 & 30.8 \\
\hline High school & 401 & 36.8 \\
\hline Secondary school & 270 & 24.7 \\
\hline Primary school & 83 & 7.6 \\
\hline \multicolumn{3}{|l|}{ Marital status } \\
\hline Married & 712 & 65.3 \\
\hline Divorced or widowed & 121 & 11.1 \\
\hline Single & 257 & 23.6 \\
\hline \multicolumn{3}{|l|}{ Male occupation } \\
\hline Worker & 107 & 25.5 \\
\hline Employee & 123 & 29.3 \\
\hline Self-employed & 134 & 31.9 \\
\hline Retired & 39 & 9.3 \\
\hline Jobless & 16 & 3.8 \\
\hline \multicolumn{3}{|l|}{ Female occupation } \\
\hline Housewife & 447 & 66.6 \\
\hline Worker & 33 & 4.9 \\
\hline Employee & 130 & 19.3 \\
\hline self-employment & 49 & 7.3 \\
\hline Retired & 12 & 1.2 \\
\hline \multicolumn{3}{|l|}{ History of chronic disease } \\
\hline Yes & 258 & 23.7 \\
\hline No & 832 & 76.3 \\
\hline
\end{tabular}

people without any chronic disease had better preventive behaviors to COVID-19. Employees' occupational group had better mean scores of preventive behaviors than workers and unemployed people $(\mathrm{p}<0.05$, Table 4$)$.

The regression analysis indicated that female gender ( $\beta=0.11)$, perceived benefits $(\beta=0.10)$, perceived barriers ( $\beta=-0.18)$, external cues to action $(\beta=0.25)$, and internal cues to action $(\beta=0.12)$ were significantly associated with COVID-19 preventive behaviors ( $\mathrm{p}<0.05)$, (Table 5).

\section{Discussion}

The Health Belief Model (HBM) proposes that the person's attitude influences health-related behavior and 
Table 3 Correlations between behavior and HBM construct

\begin{tabular}{|c|c|c|c|c|c|c|c|}
\hline & $\begin{array}{l}\text { Perceived } \\
\text { susceptibility }\end{array}$ & $\begin{array}{l}\text { Perceived } \\
\text { severity }\end{array}$ & Benefit & Barrier & $\begin{array}{l}\text { Internal Cues to } \\
\text { action }\end{array}$ & $\begin{array}{l}\text { External Cues to } \\
\text { action }\end{array}$ & behavior \\
\hline $\begin{array}{l}\text { Perceived } \\
\text { susceptibility }\end{array}$ & 1 & & & & & & \\
\hline Perceived severity & $0.31^{b}$ & 1 & & & & & \\
\hline Benefit & 0.03 & $0.23^{b}$ & 1 & & & & \\
\hline Barrier & $0.65^{b}$ & $0.16^{\mathrm{a}}$ & $-0.08^{\mathrm{a}}$ & 1 & & & \\
\hline $\begin{array}{l}\text { Internal Cues to } \\
\text { action }\end{array}$ & $0.07^{\mathrm{a}}$ & $0.26^{b}$ & $0.31^{\mathrm{b}}$ & -0.03 & 1 & & \\
\hline $\begin{array}{l}\text { External Cues to } \\
\text { action }\end{array}$ & 0.02 & $0.27^{b}$ & $0.38^{\mathrm{b}}$ & $-0.13^{b}$ & $0.59^{\mathrm{a}}$ & 1 & \\
\hline behavior & 0.04 & $0.21^{b}$ & $0.30^{\mathrm{b}}$ & $-0.20^{\mathrm{a}}$ & $0.42^{\mathrm{b}}$ & $0.47^{b}$ & 1 \\
\hline
\end{tabular}

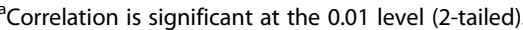

${ }^{b}$ Correlation is significant at the 0.05 level (2-tailed)

perception, i.e. changing attitudes and beliefs lead to preventive health behavior [22]. The results of the present study showed a significant positive correlation among internal/external cues to action, perceived severity and benefits, and COVID-19 preventive behaviors. Furthermore, a negative correlation was found between perceived barriers and COVID-19 preventive behaviors. In general, the present study results were in line with the results of previous studies [18, 23-26].

The present study showed that COVID-19 perceived severity in older people (aged 70+) was more than that of younger people (aged 15-29). Studies have shown that older people are at higher risk of COVID-19. Similarly, national clinical COVID-19 registries have shown a higher mortality rate among the elderly [27-29]. Jiang et al. suggested that the perceived threat to SARS preventive behavior was an important predictor of behavior change [30]. People who believe they are at high risk for the disease are more likely to engage in preventive behaviors. Therefore, educational programs should promote the perceived susceptibility and severity of COVID-19. Clark et al., 2020 concluded that age was not generally associated with voluntary compliance behaviors which was inconsistent with the results of the present study [31]. This study also indicated that women had better preventive behaviors than men and people with university-level compared to the elementary level, married people compared to single people, and people without the underlying disease had better preventive behaviors to COVID-19. Similarly, Clark et al., 2020 found that women were more likely to engage in health-related behaviors than men which was consistent with the results of the previous studies [32, 33].

The results of this study suggested that HBM almost described $27 \%$ of the variance (adjusted R2) in the COVID-19 preventive behaviors. These results are consistent with some previous studies. Mirzaei et al. identified that HBM explained $29.2 \%$ of the variance of preventive behaviors of COVID-19 [34]. In another study by Fathian-Dastgerdi, HBM constructs explained $42 \%$ of the variance of preventive behaviors [35]. Wang et al. in a study conducted in the wake of the COVID-19 pandemic in China showed that the strongest predictor of behavioral change was perceived susceptibility [1]. This may be explained due to the fact that during the early stage of the pandemic there was a more precautionary motive to take preventive actions. The present study was conducted a few months after the outbreak of COVID-19 when the level of public awareness about disease prevention had increased.

In the present study, internal/external cues to action were among the strongest stimulus needed to trigger preventive behavior, i.e., promoting the adoption of preventive behaviors by mass media, emphasizing on the sense of social responsibility, and sending notifications via cellphone and social media could promote COVID19 preventive behaviors.

The present study, also showed that perceived barriers were an essential factor that determines behavioral change. Perceived barriers were considered as an important factor for preventive behaviors. So, focusing (identifying, eliminating) on the barriers which hinder COVID-19 preparedness and response in humanitarian settings is necessary [22].

Currently, no licensed antiviral drug has been approved for the treatment of COVID-19. Quarantine (staying home) and social distancing policy have become strategic in controlling the COVID-19 pandemic. However, during quarantine, some people do not meet the health protocols requirements for various reasons. For people who violate a stay-at-home order, supportive community measures should be taken and taught. Furthermore, a more dynamic and vibrant environment at home which increases people's resilience during lockdown should be provided [36-38]. 
Table 4 Comparisons of mean scores for the HBM constructs, and preventive behaviors across demographic variables

\begin{tabular}{|c|c|c|c|c|c|c|c|}
\hline & Perceived susceptibility & Perceived severity & Benefit & Barrier & Internal Cues to action & External Cues to action & behavior \\
\hline Age & $M \pm S D$ & $\mathrm{M} \pm \mathrm{SD}$ & $\mathrm{M} \pm \mathrm{SD}$ & $\mathrm{M} \pm \mathrm{SD}$ & $M \pm S D$ & $\mathrm{M} \pm \mathrm{SD}$ & $\mathrm{M} \pm \mathrm{SD}$ \\
\hline $15-29$ & $21.2 \pm 5.5$ & $18.5 \pm 5.2$ & $16.4 \pm 3.2$ & $14.0 \pm 5.1$ & $15.8 \pm 4.2$ & $16.0 \pm 3.3$ & $30.8 \pm 5.1$ \\
\hline $30-49$ & $21.0 \pm 5.2$ & $19.5 \pm 4.3$ & $17.2 \pm 3.2$ & $13.4 \pm 5.6$ & $16.5 \pm 3.2$ & $17.1 \pm 2.9$ & $32.0 \pm 4.1$ \\
\hline 50-69 & $21.7 \pm 5.0$ & $19.7 \pm 6.2$ & $17.1 \pm 3.9$ & $15.2 \pm 5.1$ & $15.8 \pm 3.3$ & $16.5 \pm 3.0$ & $31.4 \pm 4.2$ \\
\hline$\geq 70$ & $22.2 \pm 6.4$ & $19.0 \pm 2.1$ & $16.7 \pm 2.4$ & $13.6 \pm 5.0$ & $16.6 \pm 2.2$ & $16.5 \pm 3.1$ & $33.6 \pm 3.1$ \\
\hline$P$ value (derived from ANOVA) & 0.63 & 0.022 & 0.005 & 0.01 & 0.03 & 0.001 & 0.007 \\
\hline \multicolumn{8}{|l|}{ Gender } \\
\hline Male & $21.4 \pm 5.2$ & $19.8 \pm 5.7$ & $17.0 \pm 3.2$ & $14.1 \pm 5.4$ & $16.2 \pm 3.2$ & $16.7 \pm 3.0$ & $31.2 \pm 4.5$ \\
\hline Female & $21.0 \pm 5.2$ & $18.8 \pm 4.6$ & $16.8 \pm 3.4$ & $13.3 \pm 5.2$ & $16.2 \pm 3.8$ & $16.7 \pm 3.1$ & $31.9 \pm 4.4$ \\
\hline$P$ value (derived from $t$ test) & 0.29 & 0.001 & 0.31 & 0.02 & 0.91 & 0.90 & 0.02 \\
\hline \multicolumn{8}{|l|}{ Education } \\
\hline University & $19.8 \pm 5.0$ & $19.1 \pm 3.9$ & $17.5 \pm 3.0$ & $12.2 \pm 5.2$ & $16.7 \pm 4.0$ & $17.2 \pm 2.9$ & $32.2 \pm 3.5$ \\
\hline High school & $20.9 \pm 4.8$ & $19.0 \pm 5.1$ & $16.9 \pm 3.0$ & $13.6 \pm 5.3$ & $16.1 \pm 3.5$ & $16.7 \pm 3.1$ & $31.7 \pm 4.7$ \\
\hline Secondary school & $23.0 \pm 6.1$ & $19.7 \pm 5.7$ & $16.5 \pm 3.9$ & $15.2 \pm 5.5$ & $15.6 \pm 3.4$ & $16.2 \pm 3.2$ & $31.2 \pm 4.6$ \\
\hline Primary school & $24.0 \pm 4.5$ & $19.3 \pm 3.8$ & $16.5 \pm 3.0$ & $16.0 \pm 4.9$ & $15.7 \pm 2.7$ & $15.8 \pm 2.5$ & $29.5 \pm 5.1$ \\
\hline$P$ value (derived from ANOVA) & 0.001 & 0.163 & 0.001 & 0.001 & 0.01 & 0.001 & 0.001 \\
\hline \multicolumn{8}{|l|}{ Marital status } \\
\hline Married & $23.1 \pm 7.0$ & $19.6 \pm 4.8$ & $17.1 \pm 3.2$ & $13.1 \pm 5.3$ & $16.3 \pm 3.3$ & $15.8 \pm 3.5$ & $32.2 \pm 4.0$ \\
\hline Divorced or widowed & $23.5 \pm 7.1$ & $17.9 \pm 4.5$ & $15.9 \pm 2.9$ & $14.8 \pm 5.1$ & $15.6 \pm 3.1$ & $17.1 \pm 3.0$ & $30.1 \pm 5.7$ \\
\hline Single & $21.6 \pm 6.5$ & $18.1 \pm 4.4$ & $16.4 \pm 3.4$ & $14.3 \pm 5.0$ & $15.7 \pm 4.5$ & $15.9 \pm 3.2$ & $30.3 \pm 5.3$ \\
\hline$P$ value (derived from ANOVA) & 0.001 & 0.001 & 0.001 & 0.002 & 0.001 & 0.001 & 0.001 \\
\hline \multicolumn{8}{|l|}{ Occupation } \\
\hline housewife & $21.7 \pm 5.4$ & $19.2 \pm 4.5$ & $16.6 \pm 3.4$ & $14.1 \pm 5.1$ & $16.0 \pm 3.3$ & $16.6 \pm 2.9$ & $31.9 \pm 4.1$ \\
\hline worker & $21.9 \pm 4.9$ & $19.8 \pm 3.7$ & $16.6 \pm 3.3$ & $15.2 \pm 5.9$ & $16.1 \pm 3.7$ & $16.4 \pm 3.4$ & $30.5 \pm 5.3$ \\
\hline Employee & $20.1 \pm 4.5$ & $18.9 \pm 4.6$ & $17.7 \pm 3.1$ & $11.9 \pm 5.0$ & $16.7 \pm 3.3$ & $17.2 \pm 3.0$ & $32.4 \pm 3.8$ \\
\hline self-employment & $21.1 \pm 5.3$ & $19.9 \pm 7.5$ & $16.7 \pm 3.4$ & $13.6 \pm 5.5$ & $15.9 \pm 3.1$ & $16.5 \pm 3.0$ & $31.3 \pm 4.6$ \\
\hline Retired & $21.8 \pm 7.0$ & $19.5 \pm 4.7$ & $16.6 \pm 2.4$ & $15.6 \pm 5.5$ & $16.7 \pm 2.9$ & $17.4 \pm 3.3$ & $31.2 \pm 4.6$ \\
\hline workless & $21.8 \pm 5.4$ & $19.6 \pm 3.5$ & $15.4 \pm 3.2$ & $16.3 \pm 4.4$ & $14.3 \pm 2.1$ & $14.0 \pm 2.3$ & $27.3 \pm 6.0$ \\
\hline$P$ value (derived from ANOVA) & 0.001 & 0.001 & 0.001 & 0.001 & 0.001 & 0.001 & 0.001 \\
\hline \multicolumn{8}{|l|}{ Chronic disease } \\
\hline Yes & $21.5 \pm 5.4$ & $19.1 \pm 4.9$ & $17.2 \pm 3.0$ & $14.1 \pm 5.8$ & $16.2 \pm 3.6$ & $16.7 \pm 3.2$ & $31.3 \pm 4.5$ \\
\hline No & $21.1 \pm 5.2$ & $19.6 \pm 5.4$ & $16.8 \pm 3.2$ & $13.5 \pm 5.2$ & $16.1 \pm 3.7$ & $16.8 \pm 3.0$ & $31.7 \pm 4.3$ \\
\hline$P$ value (derived from $t$ test) & 0.44 & 0.223 & 0.15 & 0.26 & 0.90 & 0.89 & 0.21 \\
\hline
\end{tabular}

Table 5 Multiple hierarchical linear regression analysis for the assessment of health beliefs and behavior

\begin{tabular}{|c|c|c|c|c|c|c|c|}
\hline & \multicolumn{2}{|c|}{ Un standardized Coefficients } & \multirow{2}{*}{$\begin{array}{l}\text { Standardized } \\
\beta\end{array}$} & \multirow[t]{2}{*}{$\mathbf{t}$} & \multicolumn{2}{|c|}{ 95\%confidence interval for B } & \multirow{2}{*}{$\begin{array}{l}P \\
\text { Value }\end{array}$} \\
\hline & B & SE & & & Lower Bound & Upper Bound & \\
\hline Age & 0.023 & 0.017 & 0.056 & 1.363 & -0.010 & 0.057 & 0.173 \\
\hline Gender (female) & 1.038 & 0.355 & 0.115 & 2.923 & 0.340 & 1.735 & 0.004 \\
\hline Married & 0.647 & 0.390 & 0.066 & 1.660 & -0.119 & 1.413 & 0.098 \\
\hline Education & 0.080 & 0.053 & 0.062 & 1.518 & -0.024 & 0.184 & 0.130 \\
\hline Perceived benefit & 0.129 & 0.051 & 0.105 & 2.562 & 0.030 & 0.229 & 0.011 \\
\hline Perceived barriers & -0.155 & 0.04 & -0.182 & -3.910 & -0.233 & -0.077 & $<0.001$ \\
\hline Perceived severity & 0.080 & 0.044 & 0.079 & 1.837 & -0.006 & 0.166 & 0.067 \\
\hline External Cues to action & 0.348 & 0.068 & 0.252 & 5.094 & 0.214 & 0.482 & $<0.001$ \\
\hline Internal Cues to action & 0.158 & 0.060 & 0.129 & 2.639 & 0.040 & 0.275 & 0.009 \\
\hline Perceived susceptibility & 0.042 & 0.043 & 0.047 & 0.985 & -0.042 & 0.127 & 0.325 \\
\hline
\end{tabular}




\section{Strengths and limitations of the study}

The cross-sectional nature of the study makes it challenging to derive causal relationships. The results might not be generalizable to all Iranian population. The results of the study might be subjected to recall bias as the participants were required to answer behavior questions over the past month.

Multi-stage and random sampling methods, trained interviewers, reliable and valid Health Belief model questionnaire, were among the strengths of the study.

\section{Conclusion}

Designing an educational intervention on the basis of HBM might be considered as a framework for the correction of beliefs and adherence to COVID-19 behavior. Health information campaigns need to (1) emphasize the benefits of preventive behaviors including avoiding the likelihood of getting a chronic disease and complications of the disease, (2) highlight the tips and advice to overcome the barriers (3) provide cues to action by means of showing various reminders in social media (4) focusing on adoption of COVID-19-related preventive behaviors, especially among men.

\section{Abbreviations}

HBM: Health Belief Model; COVID-19: Coronavirus disease 2019

\section{Supplementary Information}

The online version contains supplementary material available at https://doi. org/10.1186/s12889-021-11983-3.

Additional file 1. The questionnire used in the study to collect the data. The first part of the questionnaire included demographic characteristics. The second part of the questionnaire consisted of HBM constructs. The third part consisted of behaviors.

\section{Acknowledgments}

The researchers would like to express their gratitude to the participants and the staff of the health centers of Khuzestan, Iran. Special thanks are extended to Prof. Montazeri for his valuable comments on the method section and revisions.

\section{Authors' contributions}

MK and MA conceptualized the study and wrote the manuscript and provided the final draft. FB, BCh helped with study design. ABH,MJ, NH, FZ-A, RSh helped study implementation. All authors helped with manuscript writing. APH was the advisor of the study and critically reviewed the manuscript and made revisions. All the authors read and approved the final manuscript.

\section{Funding}

Ahvaz Jundishapur University of Medical Sciences, Ahvaz, Iran funded the study\# U-99041. The fund was spent on preparing materials such as questionnaires and data collection.

\section{Availability of data and materials}

The datasets used and/or analyzed during the current study are available from the corresponding author on reasonable request.

\section{Declarations}

Ethics approval and consent to participate

The study was approved by the Ethics Committee of Ahvaz Jundishapur University of Medical Sciences, Ahvaz, Iran (Registration No: IR.AJUMS.REC.1399.145). Written informed consent obtained from participants. For participants under 16 years of age, written consent was obtained from their parents.

Consent for publication

Not applicable.

\section{Competing interests}

The authors declared no potential conflicts of interest with respect to the research, authorship, and/or publication of this article. Dr. Marzieh Araban is a member of the editorial board of BMC Public Health.

\section{Author details}

${ }^{1}$ Social Determinants of Health Research Center, Saveh University of Medical Sciences, Saveh, Iran. ${ }^{2}$ Department of Public Health, Faculty of Health and Nutrition, Lorestan University of Medical Sciences, Khorramabad, Iran. ${ }^{3}$ Omidyeh Health Care System, Ahvaz Jundishapur University of Medical Sciences, Ahvaz, Iran. ${ }^{4}$ Department of Health Education and Promotion, School of Public Health, Ahvaz Jundishapur University of Medical Sciences, Ahvaz, Iran. ${ }^{5}$ Department of Nursing, School of Health and Welfare, Jönköping University, Jönköping, Sweden. ${ }^{6}$ Social Determinants of Health Research Center, Research Institute for Prevention of Non-Communicable Diseases, Qazvin University of Medical Sciences, Qazvin, Iran. ${ }^{7}$ Department of Biostatistics and Epidemiology, School of Public Health, Ahvaz Jundishapur University of Medical Sciences, Ahvaz, Iran. ${ }^{8}$ Department of health education and promotion, School of Health, Isfahan University of Medical Sciences, Isfahan, Iran.

Received: 23 November 2020 Accepted: 12 October 2021

Published online: 24 October 2021

References

1. Wang D, Hu B, Hu C, Zhu F, Liu X, Zhang J, et al. Clinical characteristics of 138 hospitalized patients with 2019 novel coronavirus-infected pneumonia in Wuhan, China. JAMA. 2020;323(11):1061-9. https://doi.org/10.1001/jama.2 020.1585 .

2. Rajabimajd N, Alimoradi Z, Griffiths MD. Impact of COVID-19-related fear and anxiety on job attributes: a systematic review. Asian J Soc Health Behav. 2021;4(2):51-5. https://doi.org/10.4103/shb.shb_24_21.

3. Ashraf A, Ali I, Ullah F. Domestic and gender-based violence: Pakistan scenario amidst COVID-19. Asian J Soc Health Behav. 2021;4(1):47. https:// doi.org/10.4103/shb.shb 4520.

4. Alimoradi Z, Broström A, Tsang HWH, Griffiths MD, Haghayegh S, Ohayon MM, et al. Sleep problems during COVID-19 pandemic and its' association to psychological distress: a systematic review and meta-analysis. EClinicalMedicine. 2021;36:100916. https://doi.org/10.1016/j.eclinm.2021.1 00916.

5. Huang C, Wang Y, Li X, Ren L, Zhao J, Hu Y, et al. Clinical features of patients infected with 2019 novel coronavirus in Wuhan, China. Lancet. 2020;395(10223):497-506. https://doi.org/10.1016/S0140-6736(20)30183-5.

6. Sohrabi C, Alsafi Z, O'Neill N, Khan M, Kerwan A, al-Jabir A, et al. World health organization declares global emergency: a review of the 2019 novel coronavirus (COVID-19). Int J Surg. 2020;76:71-6. https://doi.org/10.1016/j. ijsu.2020.02.034

7. Carico RR Jr, Sheppard J, Thomas CB. Community pharmacists and communication in the time of COVID-19: Applying the health belief model. Res Social Adm Pharm. 2021;17(1):1984-7. https://doi.org/10.1016/j.sapha rm.2020.03.017. Epub 2020 Mar 26. PMID: 32247680; PMCID: PMC7118622.

8. Shewasinad Yehualashet S, Asefa KK, Mekonnen AG, Gemeda BN, Shiferaw WS, Aynalem YA, et al. Predictors of adherence to COVID-19 prevention measure among communities in north Shoa zone, Ethiopia based on health belief model: a cross-sectional study. PLoS One. 2021;16(1):e0246006. https://doi.org/10.1371/journal.pone.0246006.

9. Araban M, et al. The COVID-19 pandemic: public knowledge, Attitudes and Practices in a central of Iran. J Educ Community Health. 2021. 
10. Mesri M, et al. Clinical features and outcomes of suspected and confirmed covid-19 patients in Saveh, Iran, 2020. J Mazandaran Univ Med Sci. 2021; 30(194):51-61.

11. Smith $L$, Butler $L$, Tully MA, Jacob $L$, Barnett $Y$, López-Sánchez GF, et al. Hand-washing practices among adolescents aged 12-15 years from 80 countries. Int J Environ Res Public Health. 2021;18(1):138. https://doi.org/1 0.3390/ijerph18010138

12. Glanz, K., B.K. Rimer, and K. Viswanath, Theory, research, and practice in health behavior and health education. 2008: Jossey-Bass.

13. Tadesse T, Alemu T, Amogne G, Endazenew G, Mamo E. Predictors of coronavirus disease 2019 (COVID-19) prevention practices using health belief model among employees in Addis Ababa, Ethiopia, 2020. Infect Drug Resist. 2020;13:3751-61. https://doi.org/10.2147/IDR.S275933.

14. Walrave M, Waeterloos C, Ponnet K. Adoption of a contact tracing app for containing COVID-19: a health belief model approach. JMIR Public Health Surveill. 2020;6(3):e20572. https://doi.org/10.2196/20572.

15. Coronavirus Cases. 2020 16.11.2020 [cited 2020 11.16.2020]; Available from: https://www.worldometers.info/coronavirus/country/iran/.

16. Zarei J, Dastoorpoor M, Jamshidnezhad A, Cheraghi M, Sheikhtaheri A Regional COVID-19 registry in Khuzestan, Iran: a study protocol and lessons learned from a pilot implementation. Inform Med Unlocked. 2021;23:100520. https://doi.org/10.1016/j.imu.2021.100520.

17. Bates BR, Moncayo AL, Costales JA, Herrera-Cespedes CA, Grijalva MJ. Knowledge, attitudes, and practices towards COVID-19 among Ecuadorians during the outbreak: an online cross-sectional survey. J Community Health. 2020;45(6):1158-67. https://doi.org/10.1007/s10900-020-00916-7.

18. Khazaei S, Bashirian S, Jenabi E, Barati M, Karimi-Shahanjarini A, Moeini B, et al . COVID-19 Preventive Behaviors and its Related Beliefs among Health Workers: The Role of Threat and Coping Appraisals. J Educ Community Health. 2020;7(3):221-7.

19. Papagiannis D, Malli F, Raptis DG, Papathanasiou IV, Fradelos EC, Daniil Z, et al. Assessment of knowledge, attitudes, and practices towards new coronavirus (SARS-CoV-2) of health care professionals in Greece before the outbreak period. Int J Environ Res Public Health. 2020;17(14):4925. https:// doi.org/10.3390/ijerph17144925.

20. Araban M, et al. The COVID-19 pandemic: public knowledge, Attitudes and Practices in a central of Iran. Hamadan-University-of-Medical-Sciences. 2021; 8(1):35-40.

21. Waltz, C.F., O.L. Strickland, and E.R. Lenz, Measurement in nursing and health research. 2010: Springer publishing company.

22. Glanz, K., B.K. Rimer, and K. Viswanath, Health behavior: Theory, research, and practice. 2015: John Wiley \& Sons.

23. Jahangiry L, Bakhtari F, Sohrabi Z, Reihani P, Samei S, Ponnet K, et al. Risk perception related to COVID-19 among the Iranian general population: an application of the extended parallel process model. BMC Public Health. 2020;20(1):1571. https://doi.org/10.1186/s12889-020-09681-7.

24. Keyvanara $\mathrm{M}$, et al. Evaluating self-care barriers in prevention of Covid-19 according to healthcare experts and laypersons: a mixed study. J Mazandaran University Med Sci. 2020;30(189):117-25.

25. Mo PKH, Wong CHW, Lam EHK. Can the health belief model and moral responsibility explain influenza vaccination uptake among nurses? J Adv Nurs. 2019;75(6):1188-206. https://doi.org/10.1111/jan.13894.

26. Tong KK, Chen JH, Yu EW, Wu AMS. Adherence to COVID-19 Precautionary Measures: Applying the Health Belief Model and Generalised Social Beliefs to a Probability Community Sample. Appl Psychol Health Well Being. 2020; 12(4):1205-23. https://doi.org/10.1111/aphw.12230. Epub 2020 Oct 3. PMID: 33010119; PMCID: PMC7675419.

27. Chan JF, et al. A familial cluster of pneumonia associated with the 2019 novel coronavirus indicating person-to-person transmission: a study of a family cluster. Lancet. 2020;395(10223):514-23. https://doi.org/10.1016/S014 0-6736(20)30154-9.

28. Emami A, Javanmardi F, Pirbonyeh N, Akbari A. Prevalence of underlying diseases in hospitalized patients with COVID-19: a systematic review and Meta-analysis. Arch Acad Emerg Med. 2020;8(1):e35.

29. Sun K, Chen J, Viboud C. Early epidemiological analysis of the coronavirus disease 2019 outbreak based on crowdsourced data: a population-level observational study. Lancet Digit Health. 2020;2(4):e201-8. https://doi.org/1 0.1016/S2589-7500(20)30026-1.

30. Jiang X, Elam G, Yuen C, Voeten H, de Zwart O, Veldhuijzen I, et al. The perceived threat of SARS and its impact on precautionary actions and adverse consequences: a qualitative study among Chinese communities in the United Kingdom and the Netherlands. Int J Behav Med. 2009;16(1):5867. https://doi.org/10.1007/s12529-008-9005-5.

31. Clark C, Davila A, Regis M, Kraus S. Predictors of COVID-19 voluntary compliance behaviors: an international investigation. Glob Transit. 2020;2: 76-82. https://doi.org/10.1016/j.glt.2020.06.003.

32. Santos J, Antunes L, Namorado S, Kislaya I, João Santos A, Rodrigues AP, et al. Oral hygiene habits in Portugal: results from the first health examination survey (INSEF 2015). Acta Odontol Scand. 2019;77(5):334-9. https://doi.org/10.1080/00016357.2018.1564839.

33. Suen LKP, So ZYY, Yeung SKW, Lo KYK, Lam SC. Epidemiological investigation on hand hygiene knowledge and behaviour: a cross-sectional study on gender disparity. BMC Public Health. 2019;19(1):401. https://doi. org/10.1186/s12889-019-6705-5.

34. Mirzaei A, Kazembeigi F, Kakaei H, Jalilian M, Mazloomi S, Nourmoradi H. Application of health belief model to predict COVID-19-preventive behaviors among a sample of Iranian adult population. J Educ Health Promot. 2021;10(1):69. https://doi.org/10.4103/jehp.jehp_747_20.

35. Fathian-Dastgerdi Z, Tavakoli B, Jaleh M. Factors associated with preventive behaviors of COVID-19 among adolescents: applying the health belief model. Res Soc Adm Pharm. 2021;17(10):1786-90. https://doi.org/10.1016/j. sapharm.2021.01.014.

36. Guo Q, Zheng Y, Shi J, Wang J, Li G, Li C, et al. Immediate psychological distress in quarantined patients with COVID-19 and its association with peripheral inflammation: a mixed-method study. Brain Behav Immun. 2020; 88:17-27. https://doi.org/10.1016/j.bbi.2020.05.038.

37. Konstantopoulou G, Raikou N. Clinical evaluation of depression in university students during quarantine due to covid-19 pandemic. Eur J Public Health Stud. 2020;3(1). https://doi.org/10.46827/ejphs.v3i1.65.

38. Zhu S, Wu Y, Zhu CY, Hong WC, Yu ZX, Chen ZK, et al. The immediate mental health impacts of the COVID-19 pandemic among people with or without quarantine managements. Brain Behav Immun. 2020;87:56-8. https://doi.org/10.1016/j.bbi.2020.04.045

\section{Publisher's Note}

Springer Nature remains neutral with regard to jurisdictional claims in published maps and institutional affiliations.
Ready to submit your research? Choose BMC and benefit from:

- fast, convenient online submission

- thorough peer review by experienced researchers in your field

- rapid publication on acceptance

- support for research data, including large and complex data types

- gold Open Access which fosters wider collaboration and increased citations

- maximum visibility for your research: over $100 \mathrm{M}$ website views per year

At $\mathrm{BMC}$, research is always in progress.

Learn more biomedcentral.com/submissions 